\title{
DEFORMABLE TRELLIS: OPEN CONTOUR TRACKING IN BIO-IMAGE SEQUENCES
}

\author{
M.E. Sargin, A. Altinok, K. Rose, B.S. Manjunath \\ Department of Electrical and Computer Engineering \\ University of California, Santa Barbara, CA 93106 \\ \{msargin,alphan,rose,manj\}@ece.ucsb.edu
}

\begin{abstract}
This paper presents an open contour tracking method that employs an arc-emission Hidden Markov Model (HMM). The algorithm encodes the shape information of the structure in a spatially deformable trellis model that is iteratively modified to account for observations in subsequent frames. As the open contour is determined on the trellis of an HMM, a dynamic programming procedure reduces the computational complexity to linear in the length of the structure (or contour). The method was developed for tracking general curvilinear structures, and tested on subcellular image sequences, where microtubules grow, shrink and undergo lateral motion from frame to frame. Microtubule length changes are modeled by the addition of appropriate transient and absorbing states to the HMM. Our results provide experimental evidence for the proposed algorithm's capability to track non-rigid curvilinear objects in challenging environments in terms of noise and clutter.
\end{abstract}

Index Terms - Biomedical image processing

\section{INTRODUCTION}

Automated data collection and quantification are becoming critical in biological research and directly impact a multitude of fronts, e.g., the exploration of the biochemical mechanics of devastating diseases and the general understanding of cellular machinery. However, biological content presents specific challenges. For example, fluorescence imaging, a mainstream imaging technique in live cell studies, introduces focal issues and photobleaching in addition to the typical contrast and noise problems. The experimenter has no control over the spatial distribution of objects, since the conditions must reflect natural processes as closely as possible. Furthermore, images consist of 2D projections of 3D biological structures, resulting in a mesh of objects where clutter becomes a common problem. Thus, analysis must be performed on images that are generally of poor quality.

A general problem is that of tracking the morphological changes in biological structures over time. A particular set of such structures, which appear as string-like formations in images, presents significant challenges in the presence of clutter. Such structures include blood vessels, actin filaments, microtubules, dendrites, etc. Accurate tracking, that is robust to clutter, is desirable for collecting measurements in such videos. In this work, we consider the problem of tracking curvilinear structures that exhibit length changes (growth and shortening) and morphological deformations. We formulate the

This study was funded by Center for Bioimage Informatics under grant NSF-ITR 0331697. problem as the tracking of an open contour without fixed or anchor points along the contour body.

Microtubules are filamentous structures that perform a number of important cellular functions by varying their lengths over time (Figure 1). Perturbations of the natural balance of this process are implicated in various diseases, including Alzheimer's and cancer. Microtubules are therefore the focus of intensive research efforts. Studies of microtubule dynamic instability seek to better understand patterns of growth and shortening behavior in response to various cellular conditions induced by disease or drugs. The analysis of microtubule dynamics is highly nontrivial and manifests all the challenges mentioned above. The potential impact of automated analysis has attracted much attention. For recent results see, e.g., [1, 2, 3, 4, $5,6]$. Herein we present a novel general approach and its application to the problem of tracking live cell microtubules.

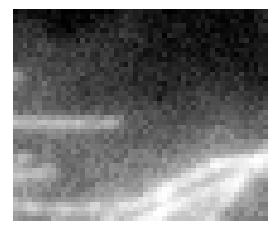

(a) Frame 1

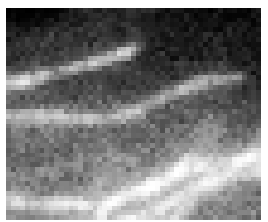

(b) Frame 10

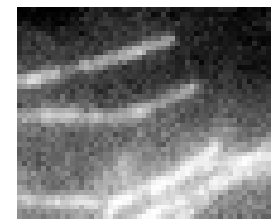

(c) Frame 12
Fig. 1. Microtubule image sequence.

\section{METHOD}

In this section we present an algorithm for tracking curvilinear structures in live cell images. The algorithm uses the estimated contour position in the previous frame as prior information, and employs an arc-emission HMM representation to properly account for observations in the current frame and to refine the contour for possible growth, shortening and lateral motion. Initialization of the tracking procedure in the first frame can be performed semi-automatically using the tracing method of [7].

\subsection{Arc-Emission HMM with Transient and Absorbing States}

The open contour at frame $t, \mathcal{C}_{t}$, is represented in a sampled format by a set of $M$ equally spaced (spacing $d_{\|}$) points indexed by $\phi \in$ $\{1,2, \ldots, M\}$. In order to capture possible deformations in the next frame, we construct a deformable trellis whose central axis is $\mathcal{C}_{t}$. The trellis is constructed by defining a set of $N$ spatial nodes that are equally spaced (spacing $d_{\perp}$ ) along the normal $\mathbf{n}_{\perp}(\phi)$ to $\mathcal{C}_{t}$ at 
each sampled location $\phi$, denoted $\mathbf{X}_{\phi}=\left\{\mathbf{x}_{\phi}(\psi) \mid \psi=1, \ldots, N\right\}$. The trellis node construction about the current contour is depicted in Fig.2, as well as its possible deformation in the next frame.

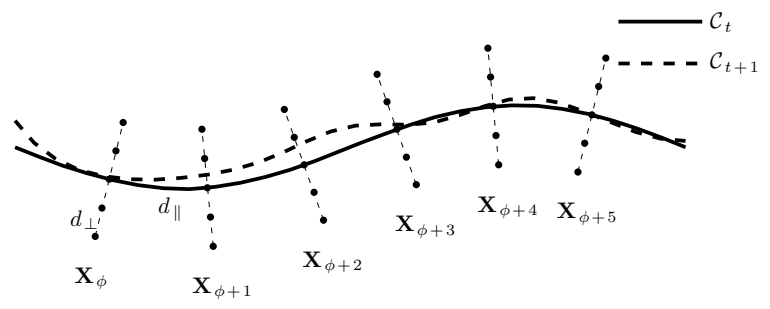

Fig. 2. Trellis node positioning about $\mathcal{C}_{t}$ covers possible deformations in $\mathcal{C}_{t+1}$. Edges between trellis nodes are not shown to avoid clutter.

Given the image at frame $t+1$ and the trellis constructed using $\mathcal{C}_{t}$, we want to find the optimal path in the trellis, i.e., the set of points $\left\{\hat{\mathbf{x}} \in \mathbf{X}_{\phi} \mid \phi=1, \ldots, M\right\}$ that will best represent $\mathcal{C}_{t+1}$. The number of possible solutions is $N^{M}$ and grows exponentially with $M$. To evaluate the quality of each possible solution we must jointly account for prior information from $\mathcal{C}_{t}$ and observation or evidence in the new frame. We propose to use the HMM, $\lambda=(\mathbf{A}, \mathbf{B}, \pi)$ as probabilistic model, and apply Viterbi decoding to find the optimal solution at computational complexity that is linear in $M$. Each state $s_{\psi}$ in $\lambda$ is associated with a point $\mathbf{x}_{\phi}(\psi)$ in $\mathbf{X}_{\phi}$.

To model length variations, and in particular shortening events, we use two auxiliary states, $s_{0}$ and $s_{N+1} . s_{0}$ is a transient state that models shortening at the initial end of the contour, and may be interpreted as the "contour has not yet begun" state. It is only possible to remain in this state or transition out of it, but impossible to return to it. $s_{N+1}$ is an absorbing state that models shortening at the other end of the contour. It is interpreted as the "contour has ended already" state. Once the system transitions to this state it will never transition out. Thus, shortening at the initial end corresponds to late transition out of state $s_{0}$, and shortening at the other end corresponds to early transition into $s_{N+1}$. We re-emphasize that $s_{0}$ and $s_{N+1}$ are transient and absorbing states, respectively, i.e., the only allowed transition into $s_{0}$ is its self transition, and the only allowed transition from $s_{N+1}$ is its self transition. Fig. 3 shows the state diagram and trellis of a simple $N=3$ system.

The HMM state transition probabilities govern the flexibility of the contour to deform, and reflect statistically extracted information about deformability prior to making any observation in frame $t+1$. The HMM emission probabilities are assigned to each state transition reflects the probability of making the observation recorded in frame $t+1$ if $\mathcal{C}_{t+1}$ passed through that specific state pair. Note that this scheme is effectively an arc-emission HMM where emission probabilities are conditioned on the state pair (transition) instead of only on the current state as is the case for "plain" HMM. This is a major departure from earlier approaches [8], which has a major practical impact as will be further explained below. We propose to incorporate with the image features observed between consecutive $\mathbf{X}_{\phi}$ 's using arc-emissions and adjust the transition matrix considering the flexibility of the $\mathcal{C}_{t}$.

Arc-emission probabilities associated with the state transition from point $\mathbf{z}_{1}=\left[x_{1}, y_{1}\right]^{T}$ to point $\mathbf{z}_{2}=\left[x_{2}, y_{2}\right]^{T}$ are determined by evaluating the evidence in the image for a contour consistent with this transition, i.e., the support $S\left(\mathbf{z}_{1}, \mathbf{z}_{2}\right)$ in the patch between the

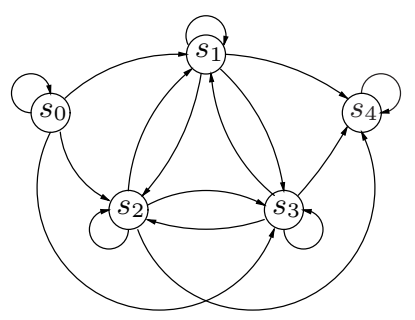

(a)

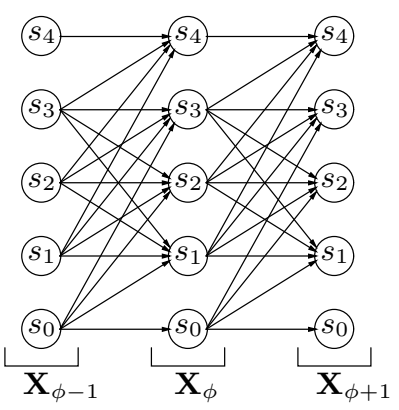

(b)
Fig. 3. (a) State transition diagram for $N=3$, and (b) corresponding HMM trellis. $s_{0}$ and $s_{4}$ are the starting and ending states, respectively.

points, which is measured by the average second order directional derivative in the direction perpendicular to the transition.

$$
S\left(\mathbf{z}_{1}, \mathbf{z}_{2}\right)=\int_{0}^{1} f\left((1-\tau) x_{1}+\tau x_{2},(1-\tau) y_{1}+\tau y_{2}, \alpha\right) d \tau
$$

where, $f(x, y, \alpha)=\mathbf{v}^{T} \mathbf{H}(x, y) \mathbf{v}, \mathbf{v}=\left[\begin{array}{ll}\cos \alpha & \sin \alpha\end{array}\right]^{T}, \alpha=$ $\arctan \left(\frac{y_{2}-y_{1}}{x_{2}-x_{1}}\right)+\frac{\pi}{2}$ and $\mathbf{H}(x, y)$ represents the Hessian matrix evaluated at point $[x, y]^{T}$.

With $S\left(\mathbf{x}_{\phi}(i), \mathbf{x}_{\phi-1}(j)\right)$ as the feature of choice, we create a gaussian model for arc-emissions in the foreground, $p(x \mid \mathcal{C})$, and background, $p(x \mid \overline{\mathcal{C}})$. The arc-emission probabilities for going from states $s_{i}$ to $s_{j}, i, j \in\{1, \ldots, N\}$, are extracted simply by evaluating the foreground arc-emission model $p(x \mid \mathcal{C})$ at $S\left(\mathbf{x}_{\phi}(i), \mathbf{x}_{\phi-1}(j)\right)$. However, the arc-emission probabilities for self transitions of $s_{0}$ and $s_{N+1}$ should reflect the evidence for background emissions for any pair of $\mathbf{x}_{\phi}(i), \mathbf{x}_{\phi-1}(j), i, j \in\{1, \ldots, N\}$. Accordingly, the arcemission probabilities, $p\left(O_{\phi} \mid s_{\phi}=i, s_{\phi-1}=j\right)$, can be formulated as follows:

$$
\begin{aligned}
& p\left(O_{\phi} \mid s_{\phi}=i, s_{\phi-1}=j\right)= \\
& \begin{cases}p\left(S\left(\mathbf{x}_{\phi}(i), \mathbf{x}_{\phi-1}(j)\right) \mid \mathcal{C}\right) & i, j \in\{1, \ldots, N\} \\
\min _{m, k \in\{1, \ldots, N\}} p\left(S\left(\mathbf{x}_{\phi}(m), \mathbf{x}_{\phi-1}(k)\right) \mid \overline{\mathcal{C}}\right) & i=j \in\{0, N+1\}\end{cases}
\end{aligned}
$$

Figure 4 illustrates a critical advantage of arc-emission HMM over the traditional state-emission HMM. In practice, and certainly in the case of microtubules, one should expect a clutter of similar objects in the vicinity. This means that although we assume some noise model for the background, we may encounter background that exhibits similar behavior to the foreground. Consider two nearby microtubules as represented in Figure 4. States that are located on either tubule will show significant evidence for a possible passage of the contour. If the emission probabilities are only conditioned on the state, there isn't sufficient penalty for jumping from one tubule to the other, which the traditional HMM will do depending on which tubule's local evidence is somewhat more pronounced at each point. (The only penalty for such skipping is provided by the state transition probabilities, but "tightening" those to solve this problem will severely compromise the flexibility of the trellis and its ability to model deformations). Arc-emission HMMs, on the other hand, allow evaluation of the local evidence for consistency with the direc- 


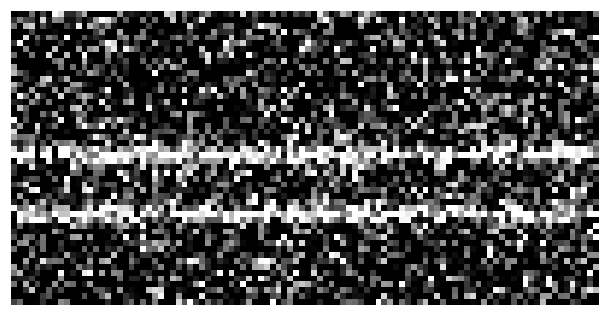

(a)

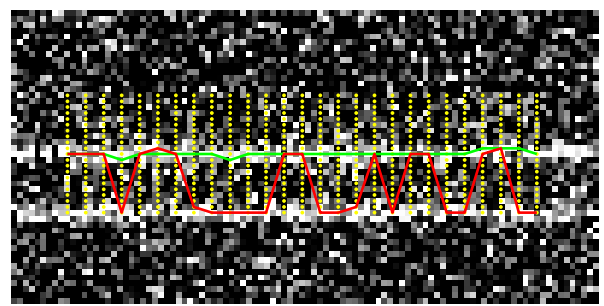

(b)

Fig. 4. (a) Synthetic image (b) Viterbi paths associated with arcemission (green) and state emission (red) HMM.

tion of the transition and can easily eliminate such confusion. This is demonstrated in the figure by the green solution.

\subsection{Extension of the Deformable Trellis}

After running the Viterbi decoding and removing the $\mathbf{X}_{\phi}$ 's associated with states $s_{0}$ and $s_{N+1}$ from the deformable trellis, we propose an algorithm to extend the deformable trellis from both ends to capture growth of the microtubule. In order to extend the deformable trellis, we make use of a graph structure at both ends of trellis which is illustrated on Figure 5. Here, we search for the best path starting from nodes $\mathbf{x}_{\phi}(\psi)$ for $\phi=1, M$ and $\psi \in[1, N]$. We also set the support of edges to be $\log \left(p\left(S\left(v_{i, j}, v_{i+1, k}\right) \mid \mathcal{C}\right)\right)$ and use the $\log$ likelihood of the trellis points, $l_{\psi}$, as the prior to satisfy stochastic constraints. In this scheme one can also use different probabilistic weights for each edge connected to the same parent node.

Suppose each vertex of the graph is represented with $v_{i, j}$ where $i$ and $j$ are the indices associated with the position of vertex in $\mathbf{n}_{\perp}$ and $\mathbf{n}_{\|}$direction. We apply Floyd-Warshall algorithm to find the solution to following equation:

$$
\mathbf{r}^{*}=\underset{\mathbf{r}}{\operatorname{argmax}} l_{r_{1}}+\sum_{i=1}^{d} \log \left(p\left(E\left(v_{i, r_{i}}, v_{i+1, r_{i+1}}\right) \mid \mathcal{C}\right)\right) .
$$

After calculating the best paths starting from all tube states at each end, we select the best path that has the minimum cost. The direction obtained by considering the root and its child node that are in the best path is considered as $\mathbf{n}_{\|}$to extend the trellis at the end.

We stop adding more points to the deformable trellis if $S\left(v_{1, r_{1}^{*}}, v_{2, r_{2}^{*}}\right)$ $\tau_{s}$. The parameter $\tau_{s}$ can be set adaptively considering the statistics of $S\left(\mathbf{x}_{\phi}\left(\frac{N+1}{2}\right), \mathbf{x}_{\phi+1}\left(\frac{N+1}{2}\right)\right)$.

Once we are done with the adding more points to the deformable trellis, the maximally likely state sequence is obtained by applying Viterbi decoding to the $\lambda$ with observation probabilities $p\left(O_{\phi} \mid s_{\phi}, s_{\phi-1}\right)$.

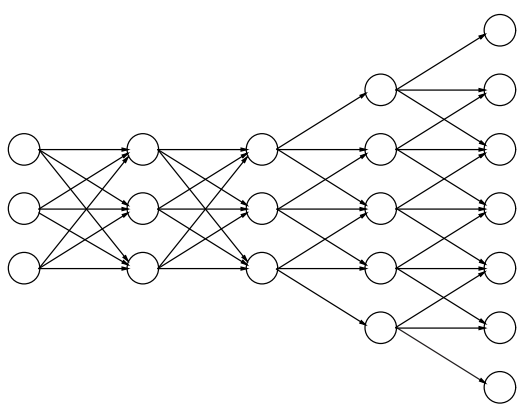

Fig. 5. Construction of the funnel for extension of the deformable trellis.

$$
\mathbf{r}^{*}=\underset{\mathbf{r}}{\operatorname{argmax}} \pi_{r_{1}} \prod_{\phi=2}^{M} p\left(O_{\phi} \mid s_{\phi}=r_{\phi}, s_{\phi-1}=r_{\phi-1}\right) a_{r_{\phi-1}, r_{\phi}}
$$

The positions of the states with indices, $\mathbf{r}^{*}$, will be the best representation of $\mathcal{C}_{t+1}$. As a predictor of the center of the deformable trellis in the next frame one can use the smoothed version of the current best path.

\section{EXPERIMENTAL RESULTS}

In our experiments we consider tracking of microtubules on live cell florescence image sequences [9] where each image sequence consists of approximately 40 frames. Four experts manually tracked microtubules by approximating their bodies with polylines in each frame. A total of 33 microtubules were collected from 8 videos, associating each tubule with 4 distinct tracks.

Assume that the mean tip position is represented with $\mathbf{u}_{\mu}=$ $E\left\{\mathbf{u}_{e_{i}}\right\}$ where $\mathbf{u}_{e_{i}}$ is the tip position of the $i$ 'th expert's polyline. Further, let $\mathbf{u}$ be the tip position of the tracking output. The experiments are conducted using both arc- and state-emission HMMs. The error measure based on the tip positions, $E\left\{\| \mathbf{t}_{\mu}-\mathbf{t}||_{2}^{2} \mid c_{2}\right\}^{0.5}$, as well as the uncertainty among the experts, $E\left\{\left\|\mathbf{t}_{\mu}-\mathbf{t}_{e_{i}}\right\|_{2}^{2}\right\}^{0.5}$, are presented in Table 1. In addition, the output of the proposed algorithm together with the ground truth from a single expert for a single image sequence is illustrated in Figure 6 and Figure 7. The tracking output on all image sequences and the ground truth are available at [10].

The distances presented in this section are in terms of pixels. During the calculation of error in tip distance, we consider the condition $c_{1} \equiv\left\|\mathbf{t}_{\mu}-\mathbf{t}\right\|_{2}<\tau_{t}=6$ on tracking success which is set by biologists. The rate of success in terms of the $c_{1}$ i.e., the number of tracks satisfy $c_{1}$ divided by the total number of tracks, is also presented in Table 1 with $p_{c_{1}}$.

\begin{tabular}{|c||c|c|}
\hline & Arc-Emission & State Emission \\
\hline \hline$p_{c_{1}}$ & 0.89 & 0.86 \\
\hline$E\left\{\left\|\mathbf{t}_{\mu}-\mathbf{t}\right\|_{2}^{2} \mid c_{2}\right\}^{0.5}$ & 4.13 & 4.43 \\
\hline$E\left\{|| \mathbf{t}_{\mu}-\mathbf{t}_{e_{i}} \|_{2}^{2}\right\}^{0.5}$ & \multicolumn{2}{|c|}{1.39} \\
\hline
\end{tabular}

Table 1. Performance of proposed tracking algorithm and the uncertainty measure among the experts. 
The majority of tracking failures were due to $C_{t+1}$ jumping to and tracking a nearby tubule in the rest of the frames. While we include these tracking failures in the rate of success, we exclude them from the tip distance statistics. Therefore, we present conditional tip distance statistics based on the condition $c_{2} \equiv\left\|\mathbf{t}_{\mu}-\mathbf{t}\right\|_{2}<5 \tau_{t}$ where $5 \tau_{t}=30$ is selected to eliminate the outliers described earlier.

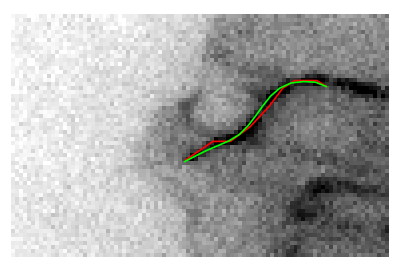

(a) Frame 5

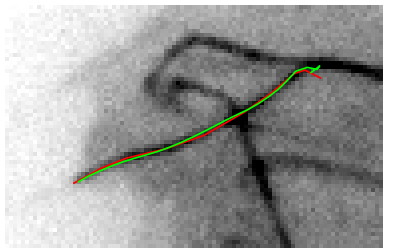

(c) Frame 36

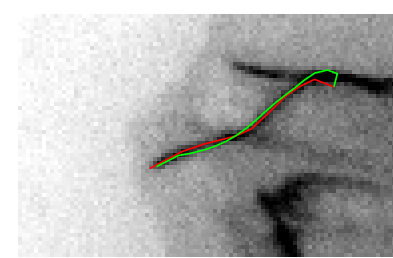

(b) Frame 15

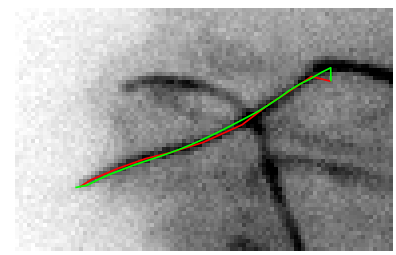

(d) Frame 41
Fig. 6. Output of the proposed algorithm (green) and the ground truth (red).

\section{CONCLUSION}

In this paper we propose an arc-emission HMM based open contour tracking algorithm. In addition to the tubule states that capture the lateral deformations, we consider auxiliary transient and absorbing states that capture the shortening of the contour. In the case of contour growth, we consider a graph structure where the edge weights are selected consistently with the arc-emissions of the HMM. Experiments reported that arc-emissions performs better than state emissions since it incorporates with the image features between consecutive $\mathbf{X}_{\phi}$ 's.

\section{REFERENCES}

[1] A. Altinok, M. El-Saban, A. J. Peck, L. Wilson, S.C. Feinstein, B. S. Manjunath, and K. Rose, "Activity analysis in microtubule videos by mixture of hidden markov models," IEEE CVPR 2006, vol. 2, pp. 1662-1669, 2006.

[2] M. El-Saban, A. Altinok, A. J. Peck, C. Kenney, S.C. Feinstein, L. Wilson, K. Rose, and B. S. Manjunath, "Automated tracking and modeling of microtubule dynamics," IEEE ISBI, pp. 10321035, 2006.

[3] S. Hadjidemetriou, J. S. Duncan, D. Toomre, and D. Tuck, "Automatic quantification of microtubule dynamics," in IEEE ISBI, 2004.

[4] S. Hadjidemetriou, D. Toomre, and J. S. Duncan, "Tracking the motion of the outer tips of microtubules," in IEEE ISBI, 2006.

[5] S. Hadjidemetriou, D. Toomre, and J. S. Duncan, "Segmentation and 3D reconstruction of microtubules in total internal reflection fluorescence microscopy (TIRFM)," in MICCAI, 2005.

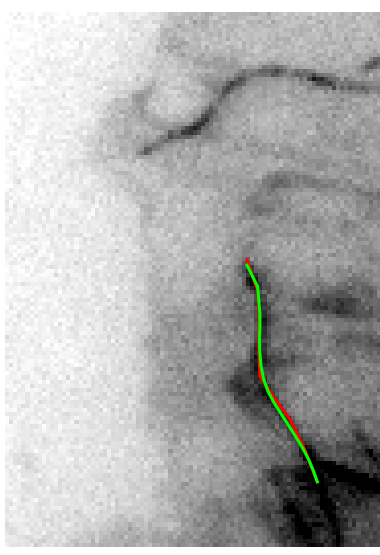

(a) Frame 5

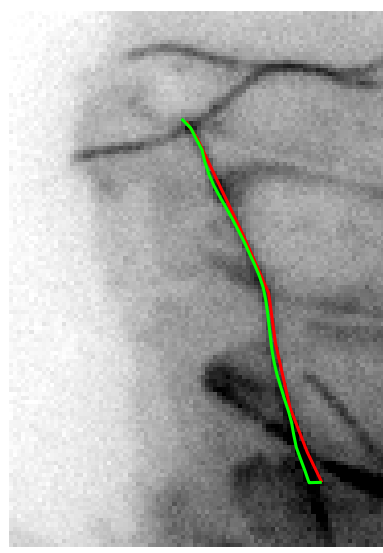

(c) Frame 24

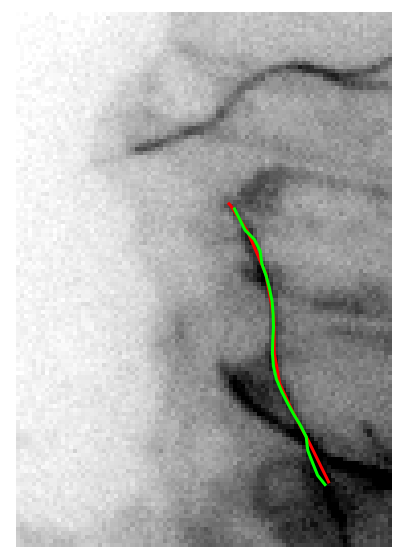

(b) Frame 12

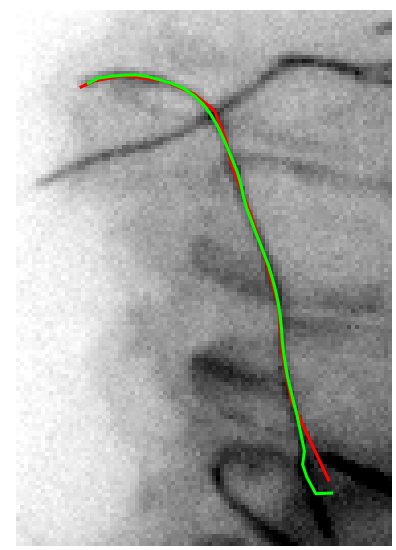

(d) Frame 41
Fig. 7. Output of the proposed algorithm (green) and the ground truth (red).

[6] M. Jiang, J. Qiang, and B. F. McEwen, "Model-based automated segmentation of kinetochore microtubule from electron tomography," in Proc. of 26th International Conf. of the Engineering in Medicine and Biology Society, 2004.

[7] M. E. Sargin, A. Altinok, K. Rose, and B.S. Manjunath, "Tracing curvilinear structures in live cell images," in IEEE International Conference on Image Processing (ICIP 07), Sep 2007.

[8] Y. Chen, Y. Rui, and T. S. Huang, "Multicue hmm-ukf for realtime contour tracking," IEEE, TPAMI, vol. 28, no. 9, pp. 1525 $-1529,2006$.

[9] K. Kamath, L. Wilson, F. Cabral, and M. A. Jordan, "ßiiitubulin induces paclitaxel resistance in association with reduced effects on microtubule dynamic instability," The Journal of Biological Chemistry, vol. 280, no. 13, pp. 12902-12907, April 2005.

[10] Tracking output videos are available at http: / / www . ece. ucsb.edu/ msargin/icassp08/. 